\title{
UNA APROXIMACIÓN AL CONCEPTO DE IDENTIDAD CULTURAL A PARTIR DE EXPERIENCIAS: EL PATRIMONIO Y LA EDUCACIÓN
}

\section{An approach to the concept of cultural identity from experiences: heritage and education}

JESÚS CEPEDA ORTEGA

GRADO DE MAESTRO EN EDUCACIÓN PRIMARIA

MÁSTER EN ECONOMÍA DE LA CULTURA Y GESTIÓN CULTURAL

Paseo de Belén, 1. Campus Miguel Delibes. 47011 Valladolid

jesus.cepeda@alumnos.uva.es

ORCID: http://orcid.org/0000-0001-7272-8111

Recibido: 27/6/2017 / Aceptado: 30/10/2017

Cómo citar: Cepeda Ortega, Jesús (2018). Una aproximación al concepto de identidad cultural a partir de experiencias: el patrimonio y la educación. Tabanque, 31. P. 244-262

DOI: https://doi.org/10.24197/trp.31.2018.244-262

Resumen: El presente artículo trata de ofrecer una aproximación al concepto de identidad cultural a través de la revisión de otros conceptos que forjan dicha idea, como son el patrimonio, la educación patrimonial y el turismo cultural. Además, se ponen de manifiesto diferentes proyectos y experiencias llevados a cabo tanto a nivel regional, en la comunidad de Castilla y León, como a nivel provincial, en la provincia de Palencia, sobre diferentes políticas de fomento de la identidad cultural.

A partir de dichas ideas, se pretende incentivar la creación de nuevos programas en las aulas de Educación Primaria y Secundaria que doten de sentido al patrimonio más próximo a los jóvenes con el objetivo de que lo sientan como propio.

Palabras clave: identidad cultural, patrimonio, educación patrimonial, turismo cultural, cultura, Educación Primaria y Secundaria.

Abstract: The present article aims to offer an approach to the concept of cultural identity through the revision of other concepts which build this idea, such as heritage, heritage education and cultural tourism. In addition, various projects and experiences carried out at regional level, in the community of Castile and Leon, and at provincial level, in the province of Palencia, regarding different promotional policies of cultural identity are shown.

Based on these ideas, it is pretended to encourage the creation of new programs in the Primary and Secondary Education framework that make sense the closest heritage to the youth with a view to assume it as their own.

Keywords: cultural identity, heritage, heritage education, cultural tourism, culture, Primary and Secondary Education. 
Sumario: 1. Introducción; 2. el patrimonio y la educación patrimonial, pilares de la creación de la identidad cultural; 3. políticas de fomento de la identidad cultural; 4. Conclusiones. Bibliografía.

Summary: 1 . Introduction; 2 . heritage and heritage education, pillars of the creation of cultural identity; 3 . policies to promote cultural identity; 4 . Conclusions. References.

\section{INTRODUCCIÓN}

La idea de este trabajo surge por el convencimiento del autor en relacionar la gestión cultural y la educación, al proceder este del segundo ámbito.

Para que el lector pueda tener una primera visión de lo que se va a encontrar en las páginas siguientes, debemos hacer un pequeño resumen del tema central que vamos a abordar. Y ese no es otro que el de la identidad cultural, un concepto poco tratado en nuestro país y que, en cambio, en otras zonas del planeta, es frecuente su uso para el reclamo de turistas.

Pero, ¿̇a qué se debe dicha utilización? Principalmente a que la identidad cultural se refiere a las características más relevantes y autóctonas de una región, de un pueblo o de una comunidad. Aquello que hace de ese territorio un lugar único, con personalidad. Y todo esto gracias al patrimonio inherente a dicho territorio, un patrimonio tangible como monumentos, obras de arte, parajes naturales, etc., y otro intangible como la lengua, las costumbres, el folclore...

Aun así, no sirve de mucho el disponer de un rico y variado conjunto de bienes patrimoniales si no se saben gestionar, preservar ni, sobre todo, valorar. Es fundamental que una comunidad sea capaz de conocer todo lo que les identifica y apreciarlo. Para ello, vemos absolutamente necesario la implantación de programas educativos en los cuales se ponga de manifiesto esta idea, con el fin de concienciar a los más jóvenes sobre el buen uso del patrimonio y de que sepan identificar los valores que este tiene para su comunidad.

Además, aunque nos hallemos ante un periodo de recesión económica, el sector cultural no sigue una evolución similar a la del resto de sectores de actividad del país. Se trata de un sector especial, que se encuentra en pleno auge y al que se le debe dar la importancia que merece, puesto que la cultura es muy necesaria para cualquier persona, para educar y formar al futuro de nuestro país.

\section{EL PATRIMONIO Y LA EDUCACIÓN PATRIMONIAL, PILARES DE LA CREACIÓN DE LA IDENTIDAD CULTURAL}


Antes de comenzar a hablar acerca de la identidad cultural, el tema central que ocupa nuestro trabajo de investigación, resulta interesante mencionar los cimientos que construyen dicho concepto, como el patrimonio y la educación patrimonial, con el objetivo de contextualizarlo para poder entender mucho mejor la segunda parte del estudio.

\subsection{Concepto de patrimonio}

Durante muchos años se ha escrito bastante acerca del carácter polisémico de la palabra patrimonio, ofreciendo numerosas ideas pero sin llegar a obtener una percepción clara sobre la que poder asentar las bases para la creación de propuestas educativas de fomento de la identidad cultural a través de los recursos patrimoniales existentes.

Por todo ello, creemos necesario realizar una revisión del concepto de patrimonio a lo largo de los últimos años:

En noviembre de 1972 se celebró la Convención sobre la protección del patrimonio mundial, cultural y natural1, donde se hacía referencia a dos conceptos diferenciados como "patrimonio cultural" y "patrimonio natural". Según el texto, dentro del primer bloque podemos encontrar monumentos, obras arquitectónicas, estructuras arqueológicas, cavernas, conjuntos arquitectónicos que integren un paisaje con un valor universal excepcional desde el punto de vista de la historia, del arte o de la ciencia; y los lugares creados por el hombre o por la propia naturaleza que posean un valor universal excepcional desde el punto de vista histórico, estético, etnológico o antropológico.

Respecto al "patrimonio natural", podemos ver que se encuentra constituido por formaciones físicas, biológicas, geológicas y fisiográficas que componen el hábitat de especies amenazadas y que presenten un valor universal excepcional y lugares naturales con un valor universal excepcional desde el punto de vista de la ciencia, de la conservación o de la belleza natural.

Sin embargo, no es hasta octubre de 2003 cuando se lleva a cabo la Convención para la salvaguardia del patrimonio cultural inmaterial2 donde se menciona el concepto de patrimonio intangible de la siguiente manera:

\footnotetext{
${ }^{1}$ Conferencia General de la Organización de las Naciones Unidas para la Educación, la Ciencia y la Cultura, en su 17ª reunión celebrada en París del 17 de octubre al 21 de noviembre de 1972.

${ }^{2}$ Conferencia General de la Organización de las Naciones Unidas para la Educación, la Ciencia y la Cultura, denominada en adelante "la UNESCO", en su 32ª reunión, celebrada en París del veintinueve de septiembre al diecisiete de octubre de 2003.
} 
"Se entiende por "patrimonio cultural inmaterial" los usos, representaciones, expresiones, conocimientos y técnicas -junto con los instrumentos, objetos, artefactos y espacios culturales que les son inherentes- que las comunidades, los grupos y en algunos casos los individuos reconozcan como parte integrante de su patrimonio cultural[...]”.

Estas definiciones no consiguen abarcar todas las perspectivas que muchos autores han expuesto en diferentes trabajos de investigación y nos dificultan la tarea de tratar de construir un concepto integrador y único. Partimos de una idea fundamental que caracteriza el patrimonio: su carácter simbólico como creador de identidades culturales. A través de esta percepción podemos comprender cuán importante es para un pueblo su pasado, sus costumbres, sus fiestas, sus restos arquitectónicos... Se demuestra en la actualidad, donde todo el mundo lucha por tener una identidad diferente que le aferre a su territorio cada vez más.

Pero entonces, ¿podríamos decir que todo vale para ser catalogado como patrimonio? Si volvemos a la Convención de 1972 vemos que solo las manifestaciones con un valor universal excepcional son consideradas como tal. Pero no todas las personas otorgarán el mismo valor estético e histórico a un monumento. Por ello, todo aquello que haya dejado una huella importante para una comunidad y sea identificativo de la misma podríamos considerarlo así.

Para resumir, nos quedamos con la definición que aporta en su tesis Sánchez (2016), refiriéndose al patrimonio como las expresiones y creaciones materiales o inmateriales realizadas por los seres humanos que le transfieren un valor personal, convirtiéndolo en "parte de su memoria". De este modo, el patrimonio refleja unos valores otorgados por el individuo "que hacen que se apropien de él como definidor de su individualidad”.

Por lo cual, consideramos el patrimonio como el pilar más grande para la construcción de una identidad cultural y el más susceptible de poder ser estudiado y abordado por proyectos educativos desde las etapas más iniciales del sistema educativo.

\subsection{Enseñanza del patrimonio como fomento de valores de los valores de identidad cultural}

La relación del patrimonio con la creación de una identidad cultural nos ofrece un gran abanico de posibilidades didácticas sobre las que poder trabajar en el 
aula. Para ello, debemos entender el patrimonio de manera global con el fin de facilitar la comprensión por parte de los alumnos de la realidad sociocultural que conlleva y los valores que representa.

A través de la elaboración de trabajos creativos acerca de bienes de su pasado, se pretende fomentar el respeto por la variedad multicultural, creando una nueva ciudadanía socialmente comprometida con sus valores identitarios, ayudando a los estudiantes a que posean una actitud crítica.

Consideramos como objetivo básico de la enseñanza del patrimonio en los centros educativos construir canales de comunicación entre las sociedades de nuestro pasado y las del presente que sean de utilidad para entender las raíces culturales que son inherentes a cada uno y poder ver los vestigios patrimoniales como testigos que nos cuenten quién vivía allí, cómo, cuándo, etc. De este modo, la necesidad de conservación de dicho patrimonio podrá crecer por sí sola en las mentes de los alumnos. En resumidas cuentas, se trata de que los estudiantes sean capaces de reconocer su propia identidad cultural y puedan difundirla de la manera más atractiva y real posible.

La metodología a emplear para el desarrollo de estrategias didácticas patrimoniales ha de basarse en la propia intervención sobre el medio físico, dando a conocer a los estudiantes aquello sobre lo que van a trabajar e investigar. Coincidimos con Cuenca (2013) en que la enseñanza del patrimonio se integraría en la rama conjunta de las Ciencias Sociales sin llegar a formar una única materia dentro del currículum de la Educación Obligatoria, con el fin de su conservación y protección como resultado de una etapa que ya nos pertenece y en la que tenemos que actuar como ciudadanos socialmente críticos y comprometidos.

Para concluir este apartado, debemos comentar que se están llevando a cabo proyectos relacionados con este tema, como es el caso del concurso escolar organizado por la Fundación Santa María la Real del Patrimonio Histórico y patrocinado por la Fundación Villalar conocido como Patrimonio Joven de Futuro, que explicaremos más adelante.

\subsubsection{El patrimonio como elemento identitario y de atractivo turístico. Concepto y evolución del turismo cultural}

Otra de las bases que componen el concepto de identidad cultural es el turismo, una práctica bastante antigua que comprende una serie de relaciones entre las personas habitantes de un lugar concreto y las personas que lo visitan, los turistas. 
Pero, ¿qué es lo que realmente atrae a los visitantes? Fundamentalmente las características tangibles de la cultura autóctona, como la gastronomía o la arquitectura tradicional, siempre y cuando se encuentren publicitadas de manera correcta. Para ello, resulta necesario desarrollar un completo plan de marketing que optimice todos los recursos identificativos de un territorio con el fin de que el turista que lo visite posea unos conocimientos previos mínimos y le incite una cierta curiosidad.

De esta manera, el turista será capaz de asimilar el patrimonio del territorio visitado, haciéndose una idea de la identidad cultural del mismo.

Asímismo, todas estas visitas repercutirán en la economía de los anfitriones de manera muy positiva, por lo que puede conllevar un fuerte estímulo para que los habitantes se preocupen por preservar y restaurar numerosos bienes patrimoniales que hasta entonces hubiesen podido estar abandonados y olvidados por completo.

Centrándonos en el tema que nos atañe, cuando hablamos de turismo cultural queremos decir que es aquel tipo de turismo alternativo, con otro tipo de actividades que se pueden realizar de manera paralela al descanso vacacional, en un territorio y clima atractivos, en el que los turistas tienen una serie de apetencias por visitar varios lugares con una mayor concreción que el mero sol y playa al que estamos acostumbrados cuando observamos las playas del Mediterráneo repletas de sombrillas.

La Carta del ICOMOS de Turismo Cultural3, en 1999, insiste en el turismo como intercambio cultural entre las sociedades actuales con el objetivo de que los turistas puedan vivir una experiencia única que les haga entender el pasado y el presente de su identidad cultural.

Por otro lado, nos resulta interesante poder caracterizar a ese turista que realiza visitas culturales. Pastor Alfonso (2003) hace alusión a tres tipos diferenciados de turistas culturales:

- Los “especialistas": Efectúan viajes de manera sistemática y regular. Constituyen el $10-15 \%$ de los visitantes.

- Los "muy motivados": El principal motivo de su viaje es el cultural. Representan entre el 30 y el $40 \%$ de los visitantes.

- Los "ocasionales": Solo efectúan actividades culturales irregularmente y no se desplazan más de 50 kilómetros para ello. Comprenden entre el 45 y el 60\% de los visitantes.

\footnotetext{
${ }^{3}$ Carta internacional sobre Turismo Cultural adoptada por el International Council On Monuments and Sites en la $12^{\mathrm{a}}$ Asamblea General en México, en octubre de 1999.
} 
Como hemos comentado al comienzo de este apartado, el patrimonio necesita un turista bien informado y documentado, que pueda comprender el significado y el valor del patrimonio que visita. Por ello, todo territorio debería tratar de conseguir que la mayor parte de los visitantes que reciban formen parte del primer o segundo grupo de turistas culturales. De esta manera, estos turistas serían capaces de observar el valor estético, histórico, espiritual, etc., propiciando un sentimiento de aprecio y respeto por el patrimonio mundial que pueda incluso originar la creación de convenios internacionales para el fomento del intercambio cultural que mencionaba la Carta del ICOMOS.

Para ofrecer una visión más cercana y directa y poder comprobar la importancia y el fuerte peso del turismo para la conservación del patrimonio, y, consecuentemente, la creación de la identidad cultural de una región, consideramos oportuno introducir algunos datos acerca del turismo cultural en España:

En el gráfico 1, podemos observar la evolución de los viajes realizados por personas que residen en España con un motivo cultural desde el año 2005 hasta 2014. Como se aprecia a primera vista, lo que más llama la atención es el acusado descenso de dichos viajes a partir del año 2011 hasta 2013, donde comienza a repuntar de nuevo para volver a situarse en valores similares a años anteriores como el de 2007.

Seguramente, esta disminución viene dada por la crisis económica de la que aún no nos hemos recuperado totalmente y que afecta de manera tardía al sector cultural. Sin embargo, los datos que podemos ver del último año disponible, nos propician una recuperación que pueda dar un respiro a todos los turistas que deseen realizar una visita cultural. 


\section{Gráfico 1: Número de viajes de residentes en España realizados por motivos} culturales

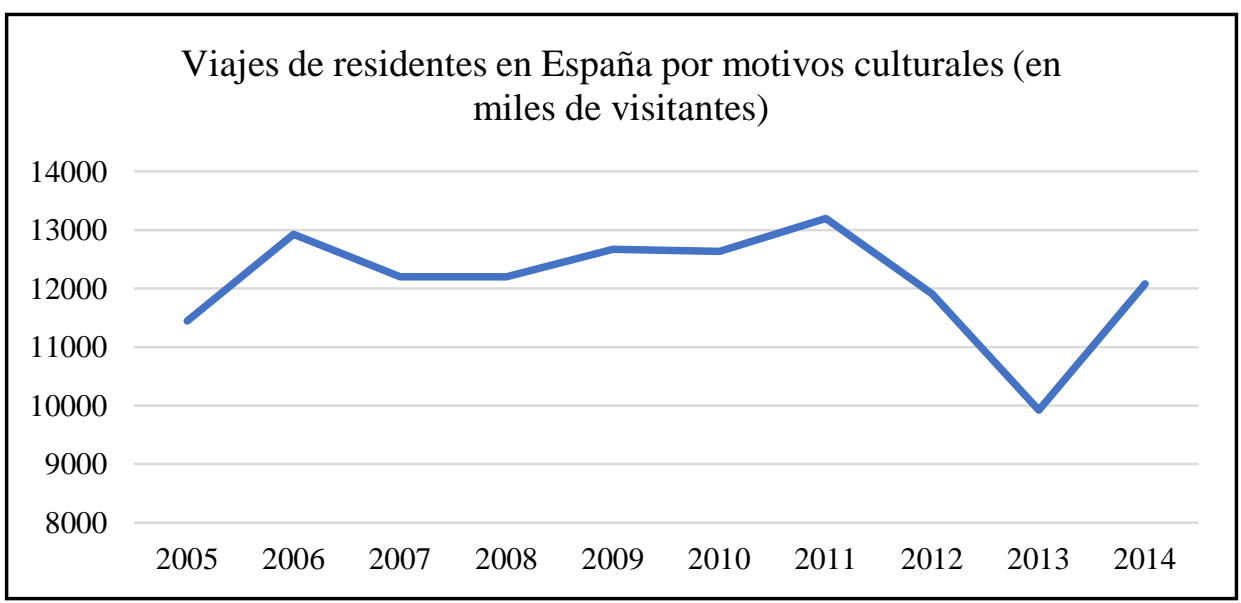

Fuente: Elaboración propia a partir de los datos de CULTURABase

Si nos fijamos en el gasto por cada viaje cultural de esos turistas, en el gráfico 2 vemos una evolución algo similar al del número de visitas. La mayor diferencia la encontramos en que la tendencia negativa aún prosigue en el año 2014 y que comenzó un año antes, existiendo una diferencia de más de $100 €$ entre el dinero que se gastaba en 2010 y 2014.

\section{Gráfico 2: Gasto por viaje realizado de residentes en España por motivos culturales}

Gasto por viaje realizado de residentes en España por motivos culturales (en euros)

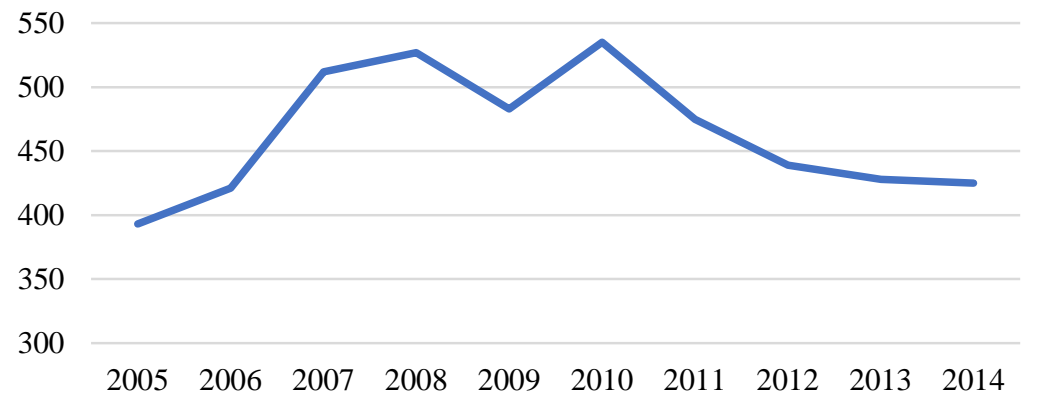

Fuente: Elaboración propia a partir de los datos de CULTURABase 
Por último, no podemos olvidar los visitantes internacionales, cuyo gasto medio en viajes culturales fue de 1074€ en el año 2014. Observando el gráfico 3, apreciamos una evolución diferente al de los anteriores gráficos, ya que la tendencia es bastante positiva y constante desde el año 2010.

\section{Gráfico 3: Entradas de turistas internacionales que realizan actividades culturales}

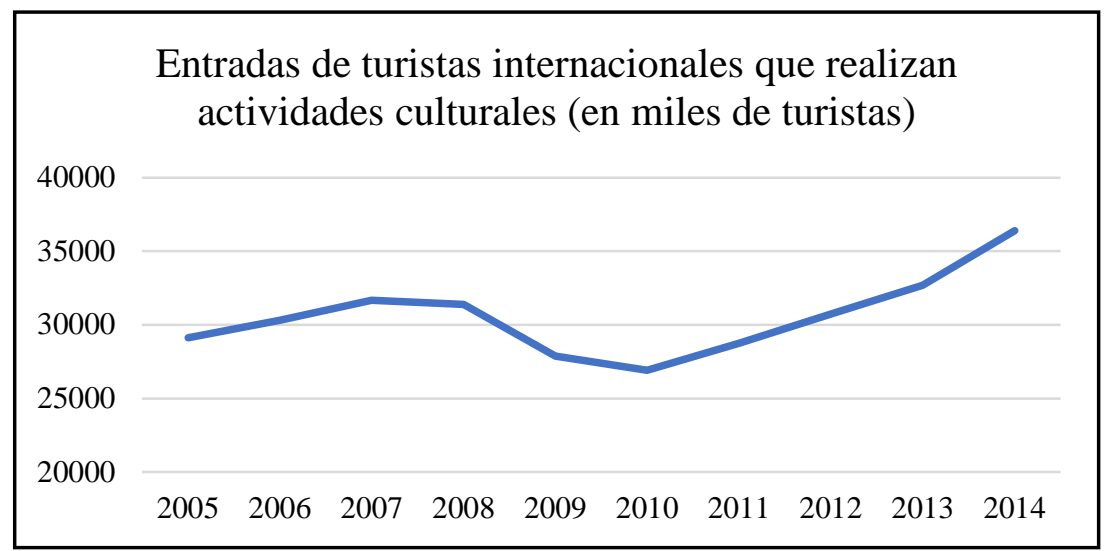

Fuente: Elaboración propia a partir de los datos de CULTURABase

Tras este pequeño análisis, podemos comprobar la gran importancia que tiene para la economía española el turismo, y más concretamente el turismo cultural. Por ello, creemos altamente necesario potenciar y preservar nuestra red patrimonial, con el objetivo de convertirse en un símbolo de identidad. La educación debe ser la principal herramienta para formar una nueva generación totalmente concienciada y con unos conocimientos que le permitan ser los artífices de la creación de una nueva época en la que el pasado y el presente convivan a través del patrimonio.

Así, hemos visto que para que cualquier persona sea capaz de valorar y diferenciar su identidad cultural y las de otras regiones y comunidades, es necesario elaborar un plan basado en la conservación y difusión del patrimonio, un patrimonio por el que el autóctono y el turista puedan entenderse, y en la creación de un nuevo turista con un mayor conocimiento del mismo. De esta manera, podemos observar cómo un turista puede tener conciencia de su identidad cultural gracias al patrimonio que le rodea y a la educación patrimonial que reciba durante sus primeros años de vida.

\subsection{Concepto de identidad cultural: cultura y territorio}


Tras esta necesaria introducción, llega el momento de comenzar a hablar sobre el hilo conductor de este estudio, la identidad cultural. Se trata de un concepto altamente repetido por numerosos autores y que sirve de tema central en múltiples convenciones internacionales culturales.

Con el fin de llegar a entenderlo y poder relacionarlo con el patrimonio y el turismo cultural, consideramos oportuno introducir una breve evolución histórica de otro concepto como es el de cultura.

\section{CULTURA}

Adam Kuper es un antropólogo sudafricano nacido en 1941, conocido por sus labores de investigación en el área de la Antropología Social, más concretamente por su estudio del concepto de cultura. En su libro "Culture: The anthropologists' account” cuenta que el origen de dicho término se remonta al siglo XVIII en Europa, precedido por otro concepto: civilización4. Todo acto contrario que no se encontraba acorde a las denotaciones cívicas era considerado salvajismo y barbarie.

Todas estas ideas continuaron evolucionando a lo largo del tiempo y se fueron conectando con las tradiciones locales, el espíritu de los ciudadanos y el territorio. Ya en el siglo XIX aparece el plural de la palabra cultura, tratando de evitar la supremacía de una única cultura y asumiendo la multiculturalidad.

Por otro lado, dicho concepto va adquiriendo un enfoque más humanista, relacionado con las costumbres y tradiciones de un pueblo y con la intelectualidad y espiritualidad personal.

Así, ya en el siglo XX, durante la Conferencia Mundial sobre las Políticas Culturales celebrada en México en el año 1982, se define cultura como "el conjunto de los rasgos distintivos, espirituales y materiales, intelectuales y afectivos que caracterizan a una sociedad o un grupo social”.

Existen numerosas definiciones, pero en general todas coinciden en una idea: "la cultura es lo que le da vida al ser humano" (Molano, 2007).

\section{IDENTIDAD CULTURAL Y TERRITORIO}

\footnotetext{
${ }^{4}$ El neologismo “civilisation" se introdujo en el Diccionario de la Academia Francesa en 1798. Anteriormente aparecía como "tecnicismo legal, haciendo referencia a la conversión de un proceso criminal en una causa civil”.
} 
Tras este breve inciso, podemos tratar de definir y contextualizar el término de identidad cultural. La identidad se encuentra ligada al desarrollo de un lugar y se va originando gracias a su evolución. Toda comunidad genera costumbres, tradiciones, leyes, etc., para crecer y avanzar a lo largo del tiempo, que componen y forman su cultura. A cambio, gracias a la cultura, dicha comunidad es capaz de continuar desarrollándose. Por ello, la identidad local de un pueblo viene caracterizada por su cultura, su desarrollo en definitiva.

Por lo tanto, podríamos decir que la identidad cultural se trata de un sentimiento de pertenencia a un colectivo social que posee una serie de características y rasgos culturales únicos, que le hacen diferenciarse del resto y por los que también es juzgado, valorado y apreciado. Aun así, no podemos confundir el concepto de identidad cultural con el de sentimiento comunitario. El primero de ellos, como acabamos de explicar, son los rasgos culturales característicos de un pueblo. En cambio, el sentimiento de comunidad es la manera que tiene cada individuo de ese pueblo de vivir y sentir dicha cultura, pero que resulta totalmente necesario para que la comunidad se mantenga cohesionada.

Todos estos elementos característicos de un grupo se expresan en manifestaciones culturales que pueden ser materiales, como monumentos, obras de arte, utensilios y herramientas..., o inmateriales, tales como la música, la danza, las fiestas, etc.

Pero si ese grupo social no valora e identifica como propio su patrimonio cultural nunca podría convertirse en una manifestación referente de su identidad, puesto que es la sociedad la causante de otorgar valor al patrimonio. De otra manera, esos vestigios culturales solo serían restos carentes de historia y simbolismo. Por todo ello, no existiría identidad cultural sin memoria.

Por lo tanto, como explica Orduna (2003), es tarea de la educación fomentar una serie de valores comunes a todas las culturas como la dignidad, el respeto, la tolerancia y la solidaridad, que propicien la creación de un clima de respeto intercultural y que conlleven un desarrollo local basado en la diversidad. De esta manera, la comunicación entre las diferentes comunidades vecinas debe ser base para la construcción de las identidades culturales de cada una de ellas.

Acabamos de comprobar la gran importancia social que tiene para un pueblo poseer un pasado sobre el que poder crecer y crear un futuro, pero no solo se trata de un beneficio para la cohesión de los habitantes, sino que también supone un desarrollo económico. Gracias a la revitalización y exaltación del patrimonio cultural de una comunidad, se puede llegar a generar una serie de actividades 
económicas relacionadas con el turismo, que repercutan de manera muy positiva en el desarrollo social del pueblo y aumente su bienestar social.

Pero esta preservación y difusión ha de llevarse a cabo de manera eficiente y original. Por ello, creemos oportuno la introducción de proyectos de fomento de la identidad cultural en las escuelas desde la Educación Primaria, aprovechando que en esas etapas el niño aún se encuentra construyendo una identidad.

A modo de ejemplo para reforzar nuestra idea, vamos a exponer de manera breve algunas experiencias de este tipo que ya se han llevado a cabo o que se encuentran vigentes en la actualidad.

\section{PolíticAs DE FOMENTO DE LA IDENTIDAD CULTURAL}

\subsection{Experiencias de programas de identidad cultural en Castilla y León}

Para contextualizar el tema del presente trabajo de investigación, vamos a tratar de exponer brevemente varios proyectos con un objetivo compartido, como es el del fomento de la identidad cultural de un territorio. Hemos elegido propuestas muy diferentes pero que persiguen el mismo fin identitario y reivindicativo con el fin de poder comprobar las diferentes formas de desarrollar este tipo de actividades.

¿Por qué estos y no otros? Básicamente por los siguientes motivos:

- Su proximidad territorial: Todos se desarrollan en la comunidad de Castilla y León y uno, más concretamente, en la localidad de Palencia.

- Su objetivo es promover y difundir la identidad cultural de un territorio.

- La metodología empleada para la puesta en práctica nos ofrecerá ideas sobre las que poder construir nuestro propio proyecto.

- Su difusión es muy distinta, por lo que nos servirá también para poder compararlos y evitar posibles errores.

\section{PATRIMONIO JOVEN DE FUTURO}

El primero de ellos se lleva a cabo en España, dentro de la comunidad de Castilla y León, con el nombre "Patrimonio joven de futuro", mencionado ya en el apartado 2.2.

Se trata de un concurso promovido por la Fundación Santa María la Real del Patrimonio Histórico en colaboración con la Dirección General de Innovación y Equidad Educativa y patrocinado por la Fundación Villalar y las Cortes de Castilla y León. 
Lleva en marcha desde el año 2001 con el título de "Los nueve secretos", aunque actualmente ha sido renovada su imagen y denominación con la fusión de la Fundación Patrimonio Histórico de Castilla y León y la Fundación Santa María la Real. Su cartel es el siguiente:

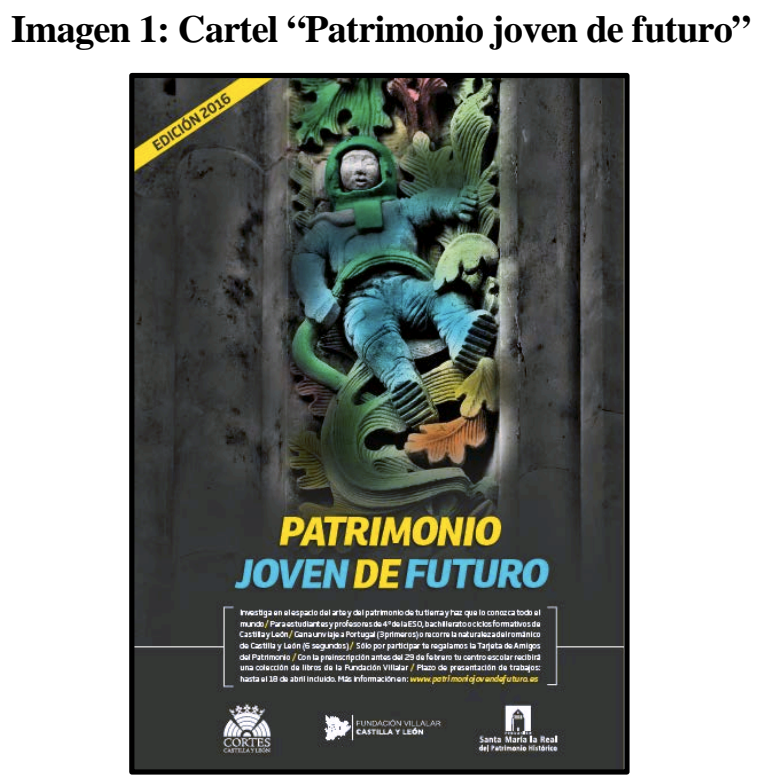

Fuente: Bases del concurso "Patrimonio joven de futuro”, Fundación Santa María la Real del Patrimonio Histórico

¿En qué consiste? Básicamente en la realización de un trabajo de investigación, documentación y propuesta de futuro, en grupos de estudiantes de $4^{\circ}$ de la ESO, bachillerato y ciclos formativos, sobre un bien que se encuentre dentro del patrimonio cultural de Castilla y León y que cumpla estas características:

- Inmueble declarado Bien de Interés Cultural (BIC).

- Mueble declarado o que se halle en el interior de un inmueble BIC.

Su principal objetivo es la sensibilización de las personas hacia su patrimonio como fuente de identidad cultural y la necesidad de su preservación y conservación.

Además, al tratarse de un concurso, existen una serie de premios y reconocimientos para los mejores trabajos presentados:

- Los tres equipos que consigan las mejores puntuaciones disfrutarán de un viaje de cinco días a Portugal.

- Los seis equipos que se queden justo por debajo de la puntuación de los tres primeros tendrán la oportunidad de realizar un viaje de fin de semana por una 
ruta cultural de Castilla y León, orientada al románico norte de la provincia de Palencia.

¿Cuáles han sido los resultados más relevantes de este proyecto? Podemos encontrar un claro ejemplo de la gran importancia que tienen las ideas de los estudiantes a través de este tipo de iniciativas en el Instituto Martínez Uribarri de Salamanca.

En el año 2006, su trabajo “La Paz y la Memoria” resultó ser uno de los tres ganadores del concurso "Los nueve secretos”. Consistía en la mejora de las instalaciones del escenario donde se llevó a cabo la batalla de Los Arapiles durante la Guerra de la Independencia Española, Sitio Histórico y Bien de Interés Cultural desde el año 1994, con el fin de adecuar las condiciones de su visita al de su valor histórico y cultural.

Gracias al apoyo del jurado, la antigua Fundación del Patrimonio Histórico puso en marcha dicho trabajo de difusión y conocimiento del campo de batalla, financiándolo con 21.685 euros junto con una subvención de la que disponía el Ayuntamiento de Arapiles de 121.000 euros.

\section{Imagen 2: Paneles informativos en el campo de batalla de Los Arapiles}

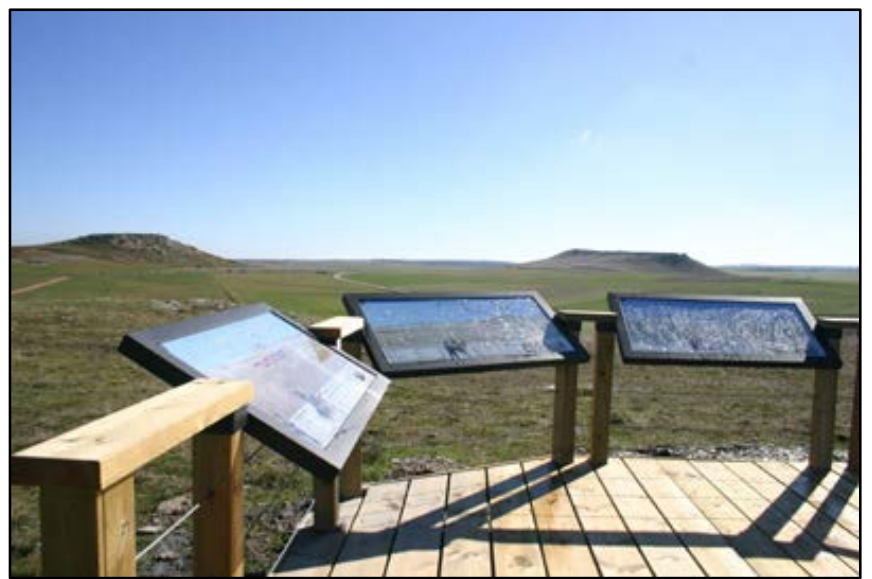

Fuente: Fundación Santa María la Real del Patrimonio Histórico

\section{ROMÁNICO ATLÁNTICO}

Otro proyecto que merece la pena mencionar se trata de un plan de intervención transfronteriza que persigue la conservación del patrimonio cultural a través de planes de la puesta en valor y de la restauración de varios templos 
románicos de varias regiones de la Península Ibérica: Zamora y Salamanca de España; Porto, Vila-Real y Bragança de Portugal.

Es una iniciativa público-privada financiada por diferentes entidades tanto españolas como portuguesas y encomendada a la Fundación Santa María la Real del Patrimonio Histórico.

El principal objetivo del proyecto es la contribución al desarrollo socioeconómico del territorio a través de acciones de conservación del patrimonio y la difusión y promoción de sus bienes. Además, se han puesto en marcha diferentes proyectos educativos, culturales y turísticos con el fin de llegar a un segmento de la población más amplio, como por ejemplo píldoras formativas para mejorar la gestión y las visitas a las iglesias.

Otro ejemplo mucho más relevante del proyecto Románico Atlántico para nuestro trabajo de investigación es la organización de talleres didácticos para grupos de niños con el objetivo de concienciar a los más jóvenes sobre la necesidad del cuidado y conservación del patrimonio que les rodea, mediante juegos y explicaciones, ya que se trata de su legado histórico y artístico.

\section{Imagen 3: Grupo de niños en un taller organizado por Románico Atlántico}

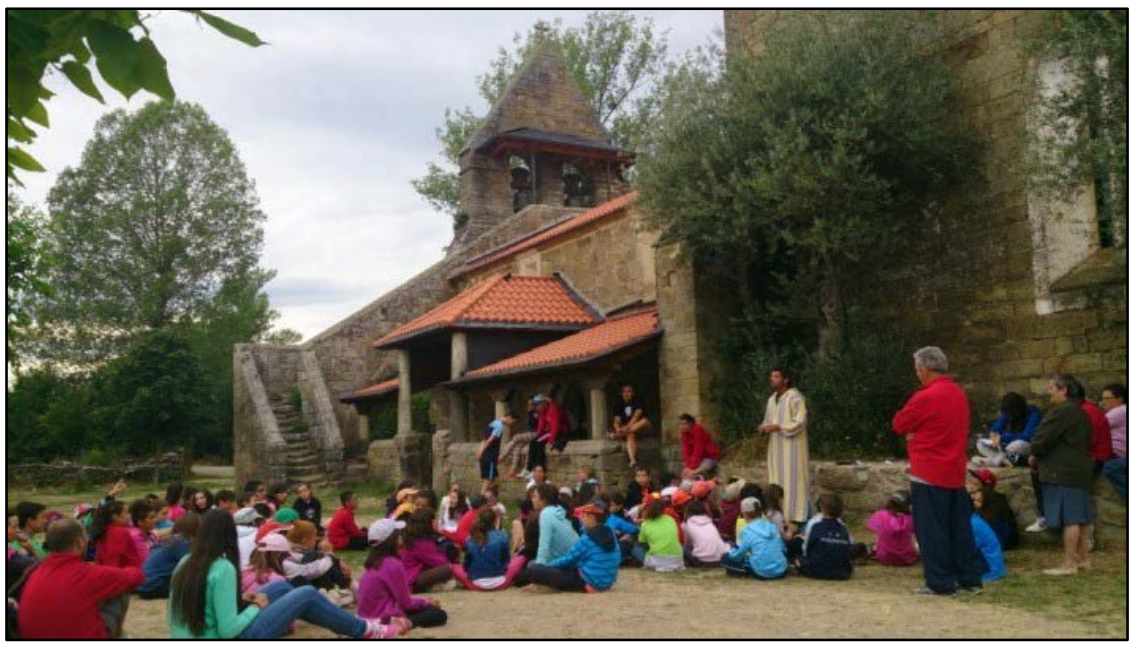

Fuente: Plan de Intervención Románico Atlántico, disponible en http://www.romanicoatlantico.org/

Este taller se trata de una de las actividades con las que el proyecto Románico Atlántico se compromete a involucrar a diversos sectores sociales, concienciándolos en la preservación y cuidado del patrimonio cultural. 


\subsection{Experiencias de programas de identidad cultural en Palencia}

\section{BARAJA CULTURAL}

La tercera experiencia que vamos a comentar es la propuesta elaborada por la Asociación de Vecinos del barrio del Cristo en la localidad de Palencia.

En el año 2013, aprovechando las celebraciones y festejos de la Romería de Santo Toribio, un grupo de vecinos del barrio del Cristo en Palencia diseñó una baraja de naipes donde quedan representados los lugares y personajes que más simbolizan y representan a dicho barrio.

La baraja fue ideada por el antropólogo César Benito e ilustrada por Ana Lorenzo, pero todo el trabajo de edición y recopilación de ideas corrió a cargo de los habitantes del lugar, así como de los propios estudiantes de los colegios del barrio.

Se puso a la venta por el módico precio de 4,50 euros, con la ilusión de que pudiera ser adquirida por cualquier persona tanto de Palencia como de cualquier otra población que se acercara al barrio para disfrutar de las actividades festivas y culturales.

\section{Imagen 4: Baraja Cultural}

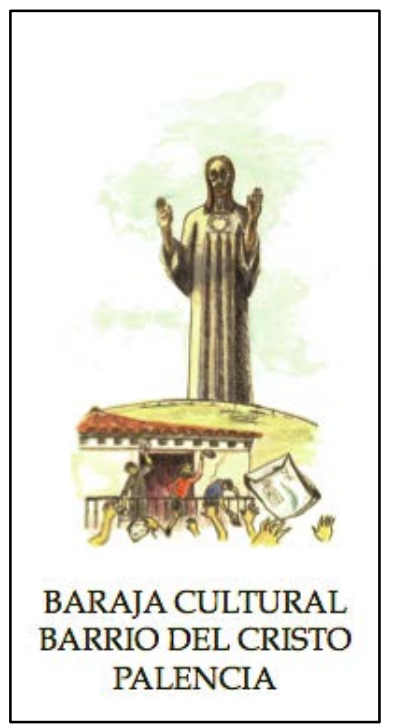

Fuente: Educación y Cultura Popular 
El objetivo principal de este proyecto tan original es el de potenciar las señas de identidad del barrio del Cristo, promoviendo el compromiso vecinal por su entorno más cercano.

Esta iniciativa se enmarca dentro de un proyecto personal de César Benito González llamado "Educación y Cultura Popular". Con él, trata de apoyar a fundaciones y entidades sin ánimo de lucro prestándoles un servicio de gestión externalizada e incluso el diseño y desarrollo de proyectos de gestión cultural.

Para terminar este apartado, tenemos que realizar un pequeño comentario con el fin de comparar estas experiencias que acabamos de exponer:

La primera de ellas se trata de una propuesta académica en la que el foco de sus destinatarios son los alumnos, mientras que esta última se encuentra más accesible a cualquier tipo de público. Sin embargo, las bases sobre las que se sustentan son más endebles en el caso de la baraja cultural, puesto que se trata de una iniciativa más popular, que abarca un territorio mucho más pequeño y que seguramente tenga y haya tenido problemas de difusión y promoción.

Teniendo en cuenta todo esto, para poder idear nuestro propio proyecto de fomento de la identidad cultural debemos identificar bien todos nuestros objetivos, así como los principales destinatarios y fijarnos un territorio lo suficientemente abarcable y con opciones de ser promocionado hacia el exterior.

\section{CONCLUSIONES}

Para concluir este artículo, debemos ser conscientes de la gran responsabilidad que tenemos como docentes con nuestros alumnos y, en definitiva, con el futuro de nuestra historia, que no es otra que nuestro patrimonio: Un patrimonio muy vivo que nos habla, que nos identifica tanto individual como colectivamente, al que hay que cuidar y gestionar de manera sostenible. Para ello, los jóvenes han de ser capaces de valorarlo, ya que sin valor no habría interés ni existiría simbología identitaria y, por consiguiente, sería imposible encontrar respeto por lo que se tiene y lo que otros tienen. Es en este momento cuando la educación debe coger las riendas para evitar ese abandono mental y físico del patrimonio tangible e intangible y colocarlo en el nivel que se merece, ya que:

- Crea una identidad cultural

- Es memoria viva

- Aporta un valor añadido

\section{BIBLIOGRAFÍA}


Benito, C. (2013). La identidad del barrio palentino del Cristo, en una Baraja Cultural. Educación y Cultura Popular. Disponible en: https://cesarbenito.wordpress.com/2013/04/15/la-identidad-del-barriopalentino-del-cristo-en-una-baraja-cultural/.

Fuentes, J. L. (2014). Identidad Cultural En Una Sociedad Plural: Propuestas Actuales Y Nuevas Perspectivas. Bordón, 66(2), 61-74. Disponible en: http://doi.org/10.13042/Bordon.2014.66204.

Fundación Santa María la Real del Patrimonio Histórico (2016). Patrimonio joven de futuro. Disponible en:

http://www.santamarialareal.org/sites/default/files/patrimoniojovendefuturo_ bases_19_1.pdf.

ICOMOS (1999). Carta internacional sobre el turismo cultural. La Gestión del Turismo en los sitios con Patrimonio Significativo. Disponible en: http://www.icomos.org/charters/tourism_sp.pdf.

Kuper, A. (2009). Culture: The anthropologists' account. Harvard University Press.

María, J., \& López, C. (2013). El papel del patrimonio en los centros educativos: hacia la socialización patrimonial. 76-96. Disponible en: https://dialnet.unirioja.es/descarga/articulo/4725269.pdf.

Molano L., O. L. (2007). Identidad cultural un concepto que evoluciona. Revista Opera, 7, 69-84. Disponible en: http://www.redalyc.org/pdf/675/67500705.pdf.

Morère Molinero, N., \& Perelló Oliver, S. (2013). Turismo Cultural: Patrimonio, museos y empleabilidad. Fundación EOI. Disponible en: http://www.turismoculturalun.org.ar/pdfs/El_turismo_Cultural_Patrimonio_ museos_y_empleabilidad.pdf.

Orduna Allegrini, G. (2003). Desarrollo local, educación e identidad cultural. Estudios sobre educación. 2003, N ${ }^{\circ}$ 4, PÁG.67- 83. Disponible en: http://dadun.unav.edu/bitstream/10171/8408/1/Estudios\%20Ee.pdf.

Rodrigo Alsina, M., Gayà, C., \& Oller, M. T. (1997). De la identidad cultural a las identidades culturales. Reflexiones, 57(1), 1-18. Disponible en: https://dialnet.unirioja.es/descarga/articulo/4796208.pdf. 
Románico Atlántico. Plan de Intervención. Disponible en:

http://www.romanicoatlantico.org/es/el-proyecto.

Sánchez Ferri, A. (2016). TESIS DOCTORAL: Memoria, identidad y comunidad:

Evaluación de programas de educación patrimonial en la Comunidad de Madrid. Universidad de Valladolid. Disponible en:

http://uvadoc.uva.es/handle/10324/16789.

Sarmiento Ramírez, I. (2007). Cultura y cultura material: aproximaciones a los conceptos e inventario epistemológico. Anales Del Museo de América, 15, 217-236. Disponible en: file:///Users/air/Downloads/Dialnet-

CulturaYCulturaMaterial-2572576\%20(1).pdf.

UNESCO. (1972). Convención sobre la protección del patrimonio mundial, cultural y natural. Disponible en: http://whc.unesco.org/archive/conventiones.pdf.

UNESCO. (1982). Conferencia mundial sobre las políticas culturales. Declaración de México Sobre Las Políticas Culturales. Disponible en: http://portal.unesco.org/culture/es/files/35197/11919413801mexico_sp.pdf/ mexico_sp.pdf.

UNESCO. (2003). Convención para la salvaguarda del patrimonio cultural inmaterial. Disponible en:

http://unesdoc.unesco.org/images//0013/001325/132540s.pdf.

Vallespir Soler, J. (1999). Interculturalismo e identidad cultural. Revista Interuniversitaria de Formación Del Profesorado. Disponible en: https://dialnet.unirioja.es/descarga/articulo/118044.pdf.

Zevallos, C. de. (2014). Estrategias didácticas para el desarrollo de la identidad cultural en educación primaria. Educación, XXIII, 25-50. Disponible en: http://revistas.pucp.edu.pe/index.php/educacion/article/view/10519. 